\title{
Downregulation of the GnT-V gene inhibits metastasis and invasion of BGC823 gastric cancer cells
}

\author{
BINBIN HUANG* ${ }^{*}$ LONGE SUN*, JIANCHUN CAO*, YUNYUN ZHANG, QIONG WU, \\ JUNJIE ZHANG, YANLI GE, LIU FU and ZHIRONG WANG
}

Department of Gastroenterology of Tongji Hospital, Tongji University School of Medicine, Shanghai, P.R. China

Received January 18, 2013; Accepted March 4, 2013

DOI: 10.3892/or.2013.2373

\begin{abstract}
Metastatic and invasive potential is a barrier to the successful treatment of gastric cancer. N-acetylglucosaminyltransferase V (GnT-V), a key enzyme catalyzing the formation of 1,6 N-acetylglucosamine (GlcNAc), has been demonstrated to display a distinct function in different types of tumors. The aim of this study was to investigate the role of GnT-V in the invasive potential of BGC823 human gastric cancer cells in vitro and the possible underlying mechanism. GnT-V was downregulated in BGC 823 cells by oligo-siRNA transfection. Cell proliferation and invasiveness were assessed by CCK-8 assay, TUNEL assay, scratch-wound assay as well as Transwell assay. The products of GnT-V, $\beta 1-6$ branching of asparagine-linked oligosaccharides, were determined by L-PHA lectin blot analysis. The expression of EGFRs, E-cadherin/vimentin and MMP-2/MMP-9 was analyzed both at the mRNA and protein levels. The results showed that downregulation of GnT-V decreased proliferation and the metastatic/invasive potential of BGC823 cells. The expression of EGFRs, E-cadherin/vimentin and MMP-9, molecules related to cancer metastasis and invasion in various tumors, were influenced correspondingly. These findings suggest that downregulation of GnT-V inhibited cell metastasis and invasion of BGC823 cells via EGFR signaling-initiated EMT phenotype and MMP-9 expression. These results provide a novel mechanism to explain the role of GnT-V in cell metastasis and invasion.
\end{abstract}

\section{Introduction}

Gastric cancer is the second most common cause of cancerrelated death in the world, and accounts for 989,600 new cases

Correspondence to: Professor Zhirong Wang, Department of Gastroenterology of Tongji Hospital, Tongji University School of Medicine, 389 Xincun Road, Shanghai 200065, P.R. China

E-mail: wzr62@yahoo.com.cn

*Contributed equally

Key words: gastric cancer, $\mathrm{N}$-acetylglucosaminyltransferase $\mathrm{V}$, metastasis, invasion and 738,000 deaths annually (1,2). Although the incidence of gastric cancer has been substantially declining for several decades, the disease is associated with a very poor prognosis, and the 5-year survival rate is $\sim 20 \%$ (3). Surgery and chemotherapy are the primary treatments. However, surgery and chemotherapy have limited value in advanced disease. In a number of cases, the disease is usually detected after invasion of the muscularis propria, and tumor metastasis serves as an impediment to successful treatment (4). Furthermore, there is an absence of molecular markers for targeted therapy (1). Thus, new perspectives in epidemiological and experimental research are important to establish novel strategies for primary prevention.

$\mathrm{N}$-acetylglucosaminyltransferase $\mathrm{V}(\mathrm{GnT}-\mathrm{V})$ is a key enzyme that catalyzes the formation of $1,6 \mathrm{~N}$-acetylglucosamine (GlcNAc) through the action of adding antennae branching structures on a common core structure of Man3GlcNAc2 in the medial-Golgi apparatus (5). Abnormalities in the expression of GnT-V are thought to be associated with tumor metastasis and invasion in various types of cancer. However, the role of GnT-V in different cancers remains controversial. In the case of colon cancer and hepatocarcinoma, for instance, high GnT-V expression is associated with a poor prognosis (6-8). In contrast, low GnT-V levels are linked to a poor prognosis in lung, bladder carcinoma and neuroblastoma patients (9-11). The role of GnT-V in gastric cancer and its invasive behavior has scarcely been studied. Tian et al (12) reported that high GnT-V expression was observed in $46 \%$ (23/50) of gastric cancer tissues and was significantly correlated with lymph node metastases, peritoneal dissemination and liver metastases, respectively. Our group previously demonstrated that high GnT-V expression was associated with a poor prognosis in patients with gastric cancer, and GnT-V was expressed at a higher level in gastric cancer BGC 823 cells than in GES-1 cells (gastric mucosal cell line) (unpublished data). Thus, it is reasonable to assume that inhibition of gastric cancer metastasis/invasion may be associated with the efficacy of repression of GnT-V.

Cancer cell metastasis/invasion is a complex process whereby tumor cells acquire the ability to dissociate from the primary lesion through activation of the epithelial growth factor receptor (EGFR) signaling pathway, through alterations in the related phenotype and the degradation of the basement membrane by matrix metalloproteinases (MMPs) consequently promoting invasion by alterating cell 
motility and growth. Aberrations in EGFR lead to the abating of downstream signaling molecules such as focal adhesion kinase (FAK), the phosphatidylinositol-3 kinase (PI3K), Ras-mitogen-activated protein kinase (Ras-MAPK) generating an anti-metastatic/invasive effect (13-18). In addition, GnT-V has been found to contribute to heparin-binding EGF-like growth factor (HB-EGF)-mediated epidermal hyperproliferation by inhibiting endocytosis of EGFRs bearing $\beta 1-6$ GlcNAc on their N-glycans (19). In addition, downregulation of $\beta 1-6$ GlcNAc branching in mammary tumor cells by overexpression of GnT-III (N-acetylglucosaminyltransferase-III), which antagonizes GnT-V activity through conformational changes in N-glycans, was found to decrease EGFR signaling (20-22). These reports highlight the importance of GnT-V mediated glycosylation of EGFR for tumor cell function. Previous reports have shown that aberrant EGFR signaling partly mediated the epithelial-mesenchymal transition (EMT) phenotype in squamous cell carcinoma and normal cells $(23,24)$. The EMT phenotype is characterized by loss of epithelial markers (E-cadherin), increased expression of mesenchymal factors (vimentin), increased migratory capacity, and resistance to apoptosis, and appears to play an important role in tumor cell metastasis/invasion (25). Furthermore, EGFR expression has also been shown to correlate with MMP expression in breast cancer cell lines and non-small cell lung cancer $(15,26)$. MMPs are a family of zinc-containing endopeptidases, among which, MMP-2 and MMP-9 are highly expressed in aggressive tumors (27-30). However, the precise mechanism of GnT-V regarding the association of EGFR signaling, EMT and MMPs in gastric cancer still remains largely unknown. Thus, given that elevated expression of GnT-V in gastric cancer and aberrant glycosylation by GnT-V are reported to modulate EGFR signaling (19), we hypothesized that downregulation of GnT-V inhibits gastric cancer metastasis/invasion through EGFR-initiated EMT phenotype and MMP-2/9 expression. Investigation of the interaction between GnT-V and EGFR expression as well as the EMT phenotype and MMPs in gastric cancer may provide insight into the underlying biological mechanism, and may offer a plausible explanation for cell migration and invasion.

In the present study, oligo-siRNA-induced RNA interference was employed to downregulate GnT-V mRNA expression in BGC823 cells (gastric cancer cell line), and the biological behavior was consequently observed. The expression levels of EGFRs, E-cadherin/vimentin and MMP-2/ MMP-9 were evaluated to further determine the underlying mechanisms.

\section{Materials and methods}

Grouping. Cells in this study were divided into four groups as listed in Table I.

Cell culture and transfection. BGC823 cells (gastric cancer cell line) were generously provided by the Cell Division of the Center Laboratory in Tongji Hospital of Tongji University, Shanghai, China. Cells were cultured in 90\% RPMI-1640 (Gibco) supplemented with $100 \mathrm{U} / \mathrm{ml}$ penicillin and streptomycin antibiotics (Gibco) and 10\% fetal bovine serum (Gibco) at $37^{\circ} \mathrm{C}$ with $5 \% \mathrm{CO}_{2}$.
The synthesized oligo-GnT-V siRNA and oligo-NC were purchased from Shanghai GenePharma Co., Ltd., Shanghai, China. The sequences are listed in Table II. Oligo-GnT-V siRNA and oligo-NC were transfected into BGC823 cells by Lipofectamine 2000 (Invitrogen, Carlsbad, CA, USA). Oligofectamine reagent for the cells $\left(6 \times 10^{4} / 3 \mathrm{ml}\right)$ consisted of $20 \mu \mathrm{M}$ oligo-pairs (22.5 $\mu \mathrm{l})$, Lipofectamine 2000 (30 $\mu \mathrm{l})$ and RPMI1640 ( $3 \mathrm{ml})$. Lipofectamine 2000 reagent was also added into the culture medium as a quality control. Transfected cells were harvested at a set time and were named BGC823/GnT-V, BGC823/NC and BGC823/ lipo cells, respectively.

Levels of GnT-V mRNA were detected by quantitative real-time reverse transcription-PCR analysis (qRT-PCR). Reverse transcription reactions used the PrimeScript RT Master Mix (Takara Biotechnology Co., Ltd., Dalian, Japan) and proceeded for $15 \mathrm{~min}$ at $37^{\circ} \mathrm{C}$ followed by $5 \mathrm{sec}$ at $85^{\circ} \mathrm{C}$ for complementary DNA (cDNA) synthesis. Real-time reactions were performed using the SYBR PrimeScript ${ }^{\mathrm{TM}}$ RT-PCR kit (Takara Biotechnology) under the following conditions: $30 \mathrm{sec}$ at $95^{\circ} \mathrm{C}$ for $1 \mathrm{cycle}, 5 \mathrm{sec}$ at $95^{\circ} \mathrm{C}, 20 \mathrm{sec}$ at $60^{\circ} \mathrm{C}$ for 40 cycles, $95^{\circ} \mathrm{C}$ for $0 \mathrm{sec}, 65^{\circ} \mathrm{C}$ for $15 \mathrm{sec}$, and $95^{\circ} \mathrm{C}$ for $0 \mathrm{sec}$ for melting curve analysis. The PCR primers (Sangon Biotech Co., Ltd., Shanghai, China) are listed in Table III. The relative mRNA expression level of GnT-V in each sample was calculated using the comparative expression level $2^{-\Delta \Delta C T}$ method. The expression of GnT-V protein was detected by western blot assay. Cells $\left(10^{7}\right)$ were harvested and lysed with ice-cold lysis buffer (RIPA and a mixture of protease inhibitors; Beyotime Institute of Biotechnology, Haimen, China). Protein concentration of the supernatant was determined by the BCA protein assay procedure. Equal amounts of proteins were separated by $10 \%$ SDS-PAGE, respectively. Proteins were then transferred to polyvinylidene difluoride membrane using a semi-dry transfer apparatus. The membranes were blocked in Tris-buffered saline (TBS) with 5\% non-fat milk for $1 \mathrm{~h}$ at room temperature, followed by incubation with the appropriate primary antibodies (1:500-diluted antibody of GnT-V, Abcam) at $4^{\circ} \mathrm{C}$ overnight. After washing in TBS-Tween 20 buffer, membranes were incubated for $2 \mathrm{~h}$ with the appropriate peroxidase-conjugated secondary antibodies, then the protein bands on the membranes were visualized using an ECL kit (Beyotime Institute of Biotechnology). The film was scanned and processed with the Odyssey Infrared Imaging system. Protein bands were quantified by Quantity One. The densitometric value of each protein band was normalized to GAPDH.

Lectin blot assay. Cells were harvested and lysed. Proteins extracted from the cells were electrophoresed on $10 \%$ SDS-PAGE, and protein-blotted PDVF membranes were prepared in exactly the same way as described for western blot analysis. After blocking with blocking solution [PBS containing $0.5 \%$ (w/v) Tween 20], the membranes were incubated with $1 \mu \mathrm{g} / \mathrm{ml} \mathrm{L-PHA} \mathrm{(Sigma)} \mathrm{for} 2 \mathrm{~h}$. Reactive bands were detected with a diluted BCIP/NBT in ExtrAvidin-AP buffer. The colorimetric reaction is normally completed within 10-20 min. The blotted proteins are colored in blue.

Cell proliferation assay (CCK-8). Cells were seeded in 96-well plates at $2 \times 10^{3}$ cells/well. At the indicated times $(0,24,48$, 
Table I. Four groups of cells in this study.

Name

Treatment

\begin{tabular}{ll}
\hline BGC823 & Cells cultured under normal condition without any treatment \\
BGC823/NC & Cells transfected with oligo-negative control (NC) siRNA and Lipofectamine 2000 reagent \\
BGC823/lipo & Cells treated with Lipofectamine 2000 reagent only \\
BGC823/GnT-V & Cells transfected with oligo-GnT-V siRNA and Lipofectamine 2000 reagent \\
\hline
\end{tabular}

Table II. Synthesized sequences.

\begin{tabular}{lc}
\hline Name & \multicolumn{1}{c}{ Sequences } \\
\hline Oligo-GnT-V siRNA F & 5'-ggcggaaauucguacagautt-3' \\
Oligo-GnT-V siRNA R & 5'-aucuguacgaauuuccgcett-3' \\
Oligo-NC F & 5'-uucuccgaacgugucacgutt-3' \\
Oligo-NC R & 5'-acgugacacguucggagaatt-3' \\
\hline
\end{tabular}

F, forward; R, reverse.

72 and 96 h), $10 \mu \mathrm{l}$ of Cell Counting Kit-8 (CCK-8; Beyotime Institute of Biotechnology) solution and $100 \mu \mathrm{l}$ RPMI-1640 plus $10 \%$ FBS were added to each well. The cells were incubated for $60 \mathrm{~min}$, and the absorbance at $450 \mathrm{~nm}$ was measured to calculate cell growth rates. Growth rate $=$ (absorbance at $450 \mathrm{~nm}$ at $\mathrm{x} \mathrm{h}$ - absorbance at $450 \mathrm{~nm}$ at $0 \mathrm{~h}) /($ absorbance at $450 \mathrm{~nm}$ at $0 \mathrm{~h}$ ).

TUNEL assay for apoptosis detection. All-trans retinoic acid (ATRA) $(80 \mathrm{mM}$ ) was used to induce apoptosis in cells. Cells were treated with $80 \mathrm{mM}$ ATRA for $24 \mathrm{~h}$ then adherent cells were fixed with $4 \%$ paraformaldehyde. Subsequently, the detection of apoptosis was preceded by TdT-mediated dUTP nick end labeling (TUNEL) assay according to the manufacturer's instructions (in situ cell death detection, Fluorescein kit, Roche). Apoptotic cells colored green were observed under a fluorescence microscope. The numbers of green fluorescence cells were counted in five different fields, selected randomly.

Cell scratch-wound assay. Cells were seeded on 24-well plates and grown to a monolayer. Wound areas were scraped using $20-\mu l$ plastic tips. At the indicated times ( 0 and $24 \mathrm{~h})$, the wound areas were photographed and the wound healing rate was calculated. Healing rate $=($ width of wound at $24 \mathrm{~h}-$ width of the wound at $0 \mathrm{~h}$ )/width of wound at $0 \mathrm{~h}$.

Cell migration assay. To evaluate cell migration capability, Transwell plates (Corning Incorporated) with a $6.5-\mathrm{mm}$ diameter filter and an $8.0-\mu \mathrm{m}$ pore size were used. Transwell chambers were inserted into a 24 -well plate. Cells $\left(1 \times 10^{5}\right)$ were plated in the upper compartment in $200 \mu 1$ of serumfree medium/chamber, and $500 \mu \mathrm{l}$ of complete medium was added to the lower wells. The chambers were incubated for $24 \mathrm{~h}$ at $37^{\circ} \mathrm{C}$ in $5 \% \mathrm{CO}_{2}$ to allow cells to migrate from the
Table III. Sequence of primers.

\begin{tabular}{|c|c|}
\hline Primers & Sequences \\
\hline GnT-V F & 5'-gaaaatggaatctgaaccctca-3' \\
\hline GnT-V R & 5'-actttgccatacacaagggact-3' \\
\hline GAPDH F & 5'-attgccetcaacgaccactt-3' \\
\hline GAPDH R & 5'-aggtccaccaccetgttgct-3' \\
\hline EGFR F & $5^{\prime}$-cgcaaagtgtgtaacggaatag-3' \\
\hline EGFR R & 5'-ccagaggaggagtatgtgtgaa-3' \\
\hline ErbB2 F & 5'-ttggtcactctgctgctgtaag-3' \\
\hline ErbB2 R & 5'-cttcattttggtagagccgaac-3' \\
\hline ErbB3 F & 5'-tgtaaggctgctgggactatg-3' \\
\hline ErbB3 R & 5'-gaacctgactgggtgacttga-3' \\
\hline ErbB4 F & 5'-ggggaataacattgcttcagag-3' \\
\hline ErbB4 R & 5'-ttaggaaggacaaggagaccaa-3 \\
\hline E-cadherin F & 5'-tgcccagaaaatgaaaaagg-3' \\
\hline E-cadherin $\mathrm{R}$ & $5^{\prime}$-gtgtatggcaatgcgttc- $3^{\prime}$ \\
\hline Vimentin F & 5'-gggacctctacgaggaggag-3' \\
\hline Vimentin R & $5^{\prime}$-cgcattgtcaacatcctgtc-3' \\
\hline MMP-2 F & $5^{\prime}$-ccactgccttcgatacac-3' \\
\hline MMP-2 R & 5'-gagccactctctggaatcttaaa-3' \\
\hline MMP-9 F & 5'-gttcccggagtgagttga-3' \\
\hline MMP-9 R & 5'-tttacatggcactgccaaagc-3' \\
\hline
\end{tabular}

F, forward; R, reverse.

upper chamber to the lower well. Cells migrating through the pores and adherent on the undersurface of the membrane were stained with Giemsa reagent. The number of cells was counted in five different fields. These fields were selected randomly.

Cell invasion assay. The cell invasion assay was performed using 24-well Transwell units with $8-\mu \mathrm{m}$ pore size polycarbonate inserts (Matrigel ${ }^{\mathrm{TM}}$ Invasion Chamber, BD Biosciences). Cells that were suspended in RPMI-1640 plus 10\% FBS were added to each upper compartment of the Transwell units. After being cultured for $24 \mathrm{~h}$, cells migrating through the Matrigel-coated polycarbonate membrane were fixed using $4 \%$ paraformaldehyde, and then stained with Giemsa reagent. The numbers of invasive cells were counted in five different fields. These fields were selected randomly. 
RNA isolation and quantitative real-time polymerase chain reaction. Total RNA was isolated from cells using the TRIzol reagent (Invitrogen). The product was reverse-transcribed into first-strand complementary DNA (cDNA). Thereafter, the expression levels of EGFR, ErbB2, ErbB3 and ErbB4, E-cadherin, vimentin, MMP-2/MMP-9, were measured using the SYBR PrimeScript ${ }^{\mathrm{TM}}$ RT-PCR Mix (Takara) according to the manufacturer's protocol. GAPDH was used to normalize the mRNA. Sequence-specific primers were designed as shown in Table III. Real-time PCR (40 cycles of denaturation at $92^{\circ} \mathrm{C}$ for $15 \mathrm{sec}$ and annealing at $60^{\circ} \mathrm{C}$ for $60 \mathrm{sec}$ ) was run on a LightCycler application system version 1.5.

Western blot assay. Cells were washed with phosphate-buffered saline (PBS) twice and lysed with ice-cold lysis buffer (RIPA) (Beyotime Institute of Biotechnology) and a mixture of protease inhibitors. The cell lysates were centrifuged at $14,000 \mathrm{x} \mathrm{g}$ for $15 \mathrm{~min}$ at $4^{\circ} \mathrm{C}$. The supernatant was collected, and the protein concentration was determined by BCA kit (Beyotime Institute of Biotechnology). Equal amounts of proteins were separated by 10 or $8 \%$ SDS-PAGE, respectively. Proteins were then transferred to PVDF membranes. The membranes were blocked in Tris-buffered saline (TBS) with $5 \%$ non-fat milk for $1 \mathrm{~h}$ at room temperature, followed by incubation with the appropriate primary antibodies (a 1:500 dilution of the antibody for MMP-2; a 1:5,000 dilution of the antibody for MMP-9; a 1:1,000 dilution of the antibody for E-cadherin; a 1:200 dilution of the antibody for vimentin; a 1:500 dilution of the antibodies of EGFR, ErbB2, ErbB3 and ErbB4) at $4^{\circ} \mathrm{C}$ overnight. All antibodies were purchased from Abcam Co., Ltd., Cambridge, MA, USA. After washing in TBS-Tween 20 buffer, membranes were incubated for $2 \mathrm{~h}$ with the appropriate peroxidase-conjugated secondary antibodies. After washing in TBS-Tween 20 buffer, the protein bands on the membranes were visualized using an ECL kit. The film was scanned and processed with Odyssey Infrared Imaging system. Protein bands were quantified by Quantity One. The densitometric value of each protein band was normalized to GAPDH.

Statistical analysis. Data are presented using means \pm SD. Statistical comparisons of groups were performed using one-way analysis of variance (ANOVA), and statistical significance was defined as $\mathrm{P}<0.05$. All the analyses were performed using SPSS 13.0 software.

\section{Results}

Downregulation of the GnT-V gene in BGC823 cells. We first explored the effect of oligo-GnT-V siRNA on GnT-V expression in BGC 823 cells. After being transfected by the constructed oligo-GnT-V siRNA, BGC823 cells were harvested at 24 and $48 \mathrm{~h}$ for future use. The result showed that the expression levels of GnT-V mRNA and protein in BGC823/GnT-V cells were decreased by $33.87 \pm 0.01$ and $35.69 \pm 2.67 \%$ when compared to the BGC823 cells $(\mathrm{P}<0.05)$ (Fig. 1). Thus, the BGC823/GnT-V cells were used in all subsequent experiments.

$\beta 1-6$ branched $N$-oligosaccharides are decreased in the BGC823 GnT-V cells. $\beta 1-6$ branching of asparagine-linked
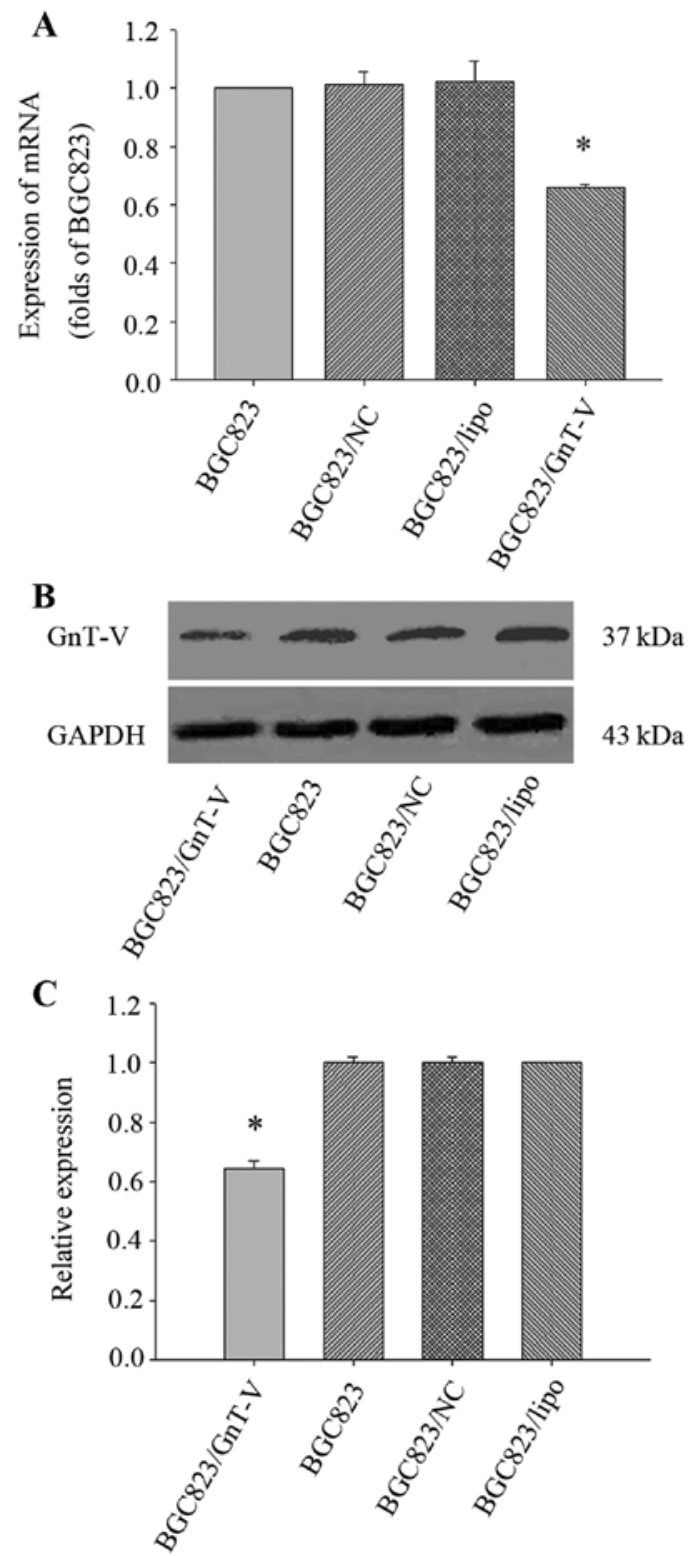

Figure 1. Downregulation of GnT-V expression in BGC823 cells. (A) The oligo-GnT-V siRNA, oligo-NC and lipo reagent were transfected into BGC823 cells. The transfected cells (BGC823/GnT-V, BGC823/NC and BGC823/ lipo) were obtained at 24 and $48 \mathrm{~h}$. Effect of oligo-GnT-V siRNA on the expression of GnT-V mRNA was determined by qRT-PCR. The expression of GnT-V mRNA in BGC823/GnT-V cells was decreased by $33.87 \pm 0.01 \%$ when compared to that in the BGC823 cells. GnT-V mRNA expression in BGC823 cells, BGC823/NC cells and BGC823/lipo cells exhibited no significant differences $(\mathrm{P}>0.05)$. (B) Effect of oligo-GnT-V siRNA on the expression of GnT-V protein was determined by western blot analysis. (C) Protein bands were quantified by Quantity One. The densitometric value of each protein band was normalized to GAPDH. The results are shown on a bar diagram. The GnT-V protein in BGC823/GnT-V cells was decreased by $35.69 \pm 2.67 \%$ compared with that in the BGC823 cells. *Statistical significance.

oligosaccharides, which are the products of GnT-V, have been shown to correlate with tumor grade and metastasis. We verified here that GnT-V suppression contributed to the reduction in $\beta 1-6$ branched N-oligosaccharides in the BGC823/GnT-V cells (Fig. 2). We performed lectin blot analysis on total cellular proteins using L-PHA, which preferentially binds to GlcNAc residues on $\beta 1-6$ branches of tri- or tetra-antennary sugar chains. This analysis showed that GnT-V catalyzed 


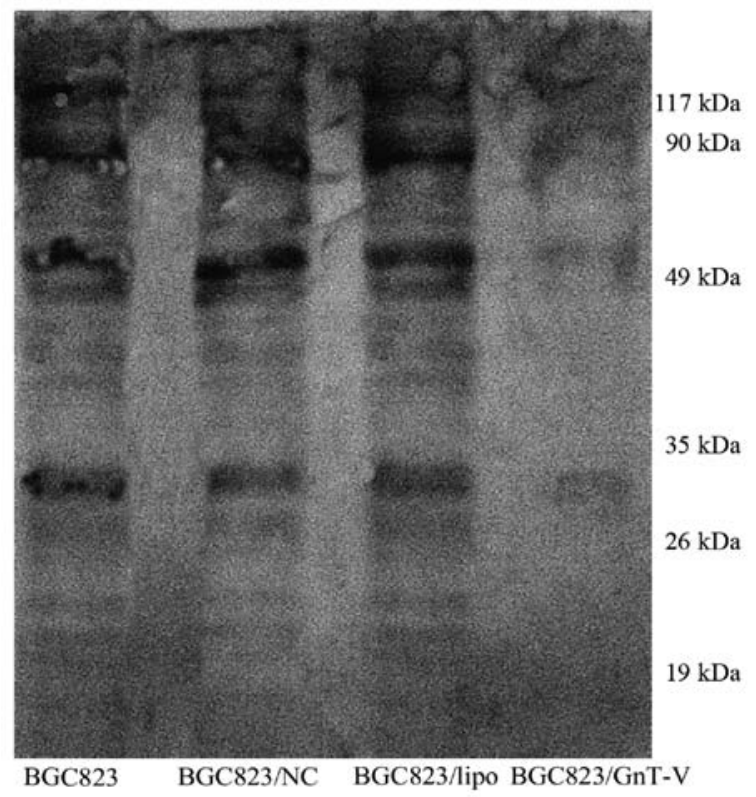

Figure 2. Lectin blot analysis with L-PHA. The amounts of $\beta 1-6$ branched $\mathrm{N}$-oligosaccharides were determined by L-PHA staining. The blotted $\beta 1-6$ branched structure is shown.

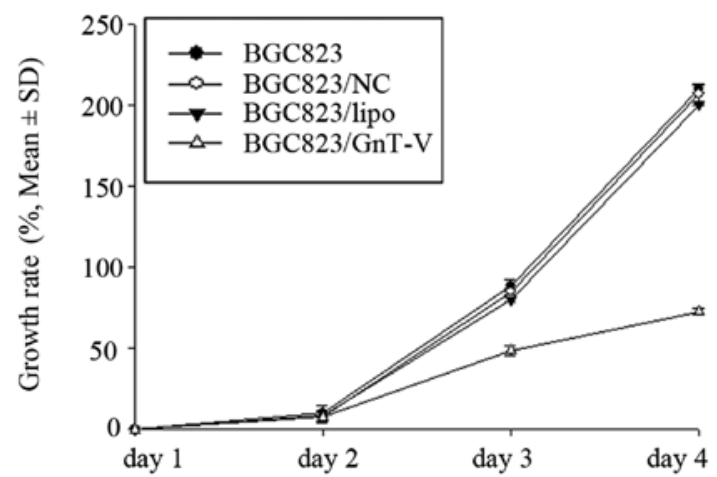

Figure 3. Comparison of the growth rates of BGC823/GnT-V, BGC 823 , BGC823/NC and BGC823/lipo cells. The numbers of BGC823/GnT-V, BGC823/NC and BGC823/lipo cells were counted over a 4-day period. Growth rate $=($ cell counts at day $\mathrm{x}-$ cell counts at day 1$) /$ cell counts at day 1 $\mathrm{x} 100 \%$.

specific glycosylation to target glycoproteins, of which major molecular sizes were $\sim 10-120 \mathrm{kDa}$. The result suggests that target substrates of GnT-V were repressed efficiently.

Proliferation of BGC823 cells is inhibited by downregulation of $G n T-V$. A previous study showed that downregulation of GnT-V induced significant growth suppression in a nasopharyngeal carcinoma cell line (31). We aimed to ascertain whether downregulation of GnT-V in gastric cancer cells contributes to inhibition of cell proliferation. We addressed this question using the CCK-8 assay to characterize the growth ability and TUNEL assay to evaluate the apoptosis rates induced by ATRA in BGC823 cells. As shown in Fig. 3, the growth rates of the BGC823/GnT-V cells were lower than those of BGC823, BGC823/NC and BGC823/lipo cells over a 4-day period. TUNEL assay revealed that the apoptosis rates of the
$\mathbf{A}$

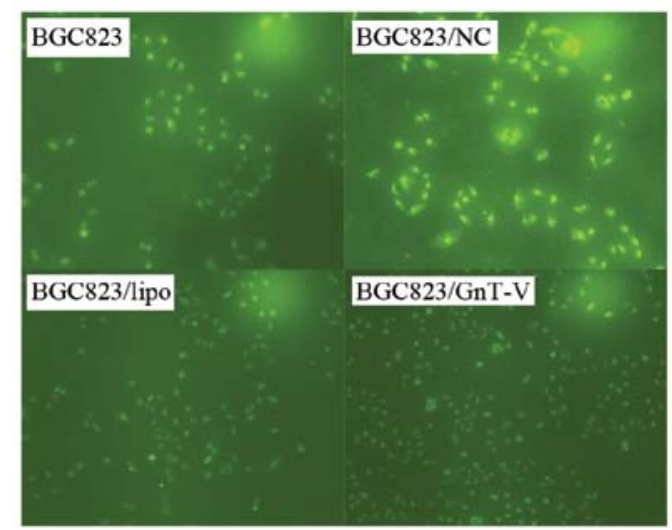

B

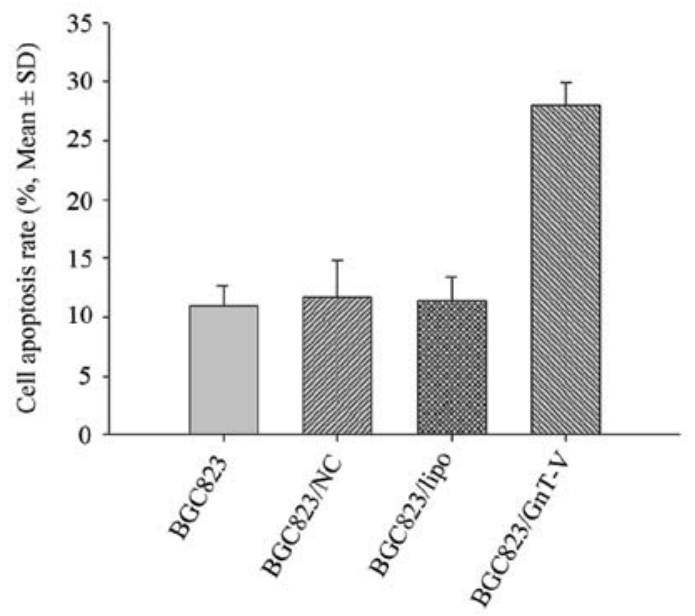

Figure 4. Cell apoptosis evaluation by TUNEL assay. (A) After cells were treated with $80 \mathrm{mM}$ ATRA for $24 \mathrm{~h}$ the adherent cells were stained using a TUNEL kit and observed under a fluorescence microscope. (B) The number of cells with green fluorescence in each group of 100 cells was counted to represent the apoptotic cells. The results are displayed as a bar diagram.

BGC823/GnT-V, BGC823, BGC823/NC and BGC823/lipo cells following treatment with $80-\mathrm{mM}$ ATRA for $24 \mathrm{~h}$ were $27.97 \pm 1.96,11.00 \pm 1.67,11.24 \pm 3.19$ and $11.39 \pm 2.08 \%$, respectively $(\mathrm{P}<0.05)($ Fig. 4), which suggests that BGC823/GnT-V cells are more susceptible to apoptosis induced by ATRA. These results showed that downregulation of GnT-V inhibited the proliferation of BGC823 cells.

Migration and invasion of BGC823 cells are inhibited by downregulation of GnT-V. The scratch-wound and Transwell migration assays were applied to detect cell migration ability. Wound healing rates of the BGC823, BGC823/NC and BGC823/lipo cells were higher than that of the BGC823/GnT-V cells at $24 \mathrm{~h}$ (Fig. 5A and B). The result of the Transwell migration assay was consistent with that of the scratch-wound assay. More BGC823, BGC823/NC and BGC823/lipo cells migrated through the membrane of the Transwell unit than BGC823/GnT-V cells $(\mathrm{P}<0.05)$ (Fig. 5C and D).

The invasive ability of the cells was determined by a Matrigel-coated Transwell assay. Fig. 6 shows that less BGC 823/GnT-V cells penetrated the Matrigel-coated membrane when compared with the number of invading BGC823, BGC823/NC and BGC823/lipo cells $(\mathrm{P}<0.05)$. 
A
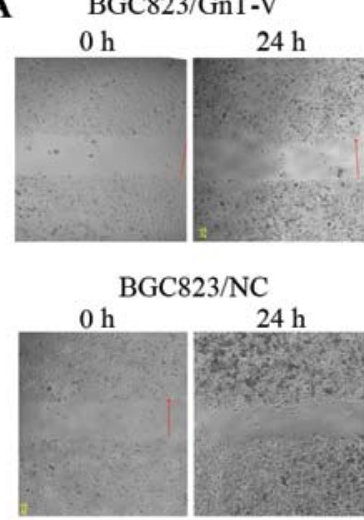

B

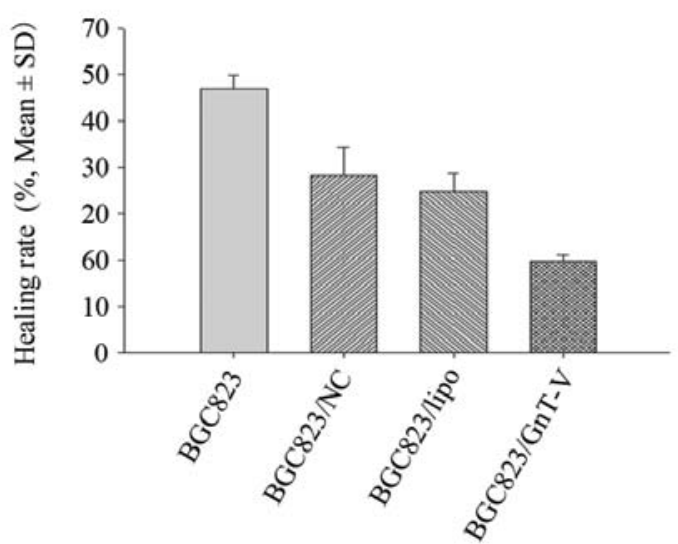

C

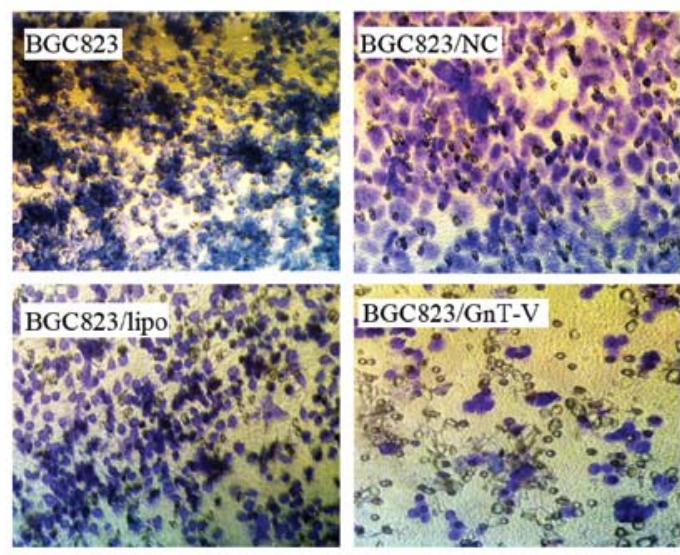

D

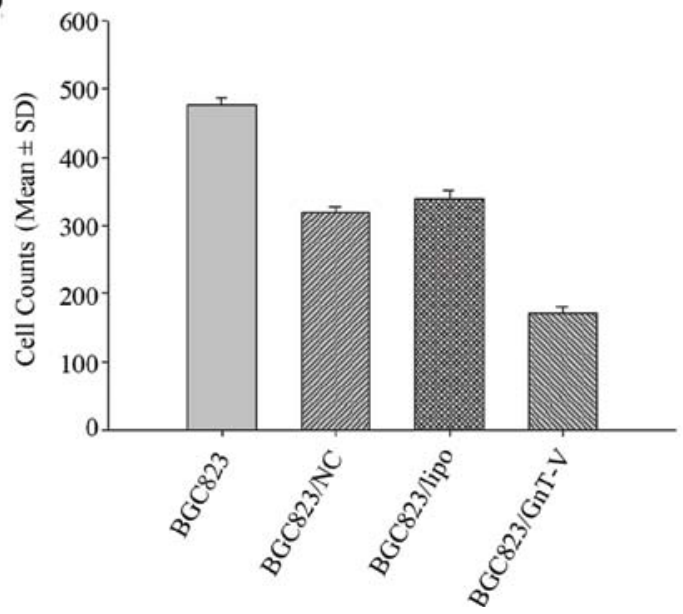

Figure 5. Cell migration ability assays. (A) A wound area was scraped using a $20-\mu 1$ plastic tip. The wound areas were photographed at 0 and $24 \mathrm{~h}$ (magnification, $\mathrm{x} 100$ ). (B) The wound healing rates are displayed on a bar diagram. The healing rate $=($ the width of the wound at $24 \mathrm{~h}-$ the width of the wound at $0 \mathrm{~h}$ )/the width of the wound at $0 \mathrm{~h}$. Data are presented as means \pm SD. (C) Using a 24-well Transwell units with $8-\mu \mathrm{m}$ pore size polycarbonate inserts, cells were spread on the polycarbonate membrane. After being cultured under the action of an inducer (cultured medium RMPI-1640 with $20 \%$ FBS) for 24 h, cells migrating through the polycarbonate membrane were fixed by paraformaldehyde, stained with Giemsa reagent and counted in five different fields. These fields were selected randomly. (D) The number of migrated cells are displayed in a bar diagram.

Expression of invasion-related factors in the BGC823/GnT-V cells. To elucidate the mechanism by which GnT-V downregulation affects EGFR-mediated aberration of the EMT phenotype and MMP-9/MMP-2 expression, the expression levels of EGFRs, E-cadherin/vimentin and MMP-2/MMP-9 were investigated. A different expression pattern for EGFR/ErbB2 and ErbB3/ErbB4 was found in the cells. EGFR and ErbB2 were decreased in the BGC823/GnT-V cells both at the mRNA and protein levels. In contrast, ErbB3 in the BGC823/GnT-V cells showed no difference when compared with the other cells both at the mRNA and protein levels. Yet, ErbB4 protein in the BGC823/GnT-V cells was deceased (Fig. 7). E-cadherin and vimentin are important regulatory markers for EMT. We found that the E-cadherin expression level was significantly higher in the BGC823/GnT-V cells, and vimentin expression was lower in the BGC823/GnT-V cells (Fig. 8). MMP-9 expression was decreased in the BGC823 GnT-V cells both at the protein and mRNA levels (Fig. 9). No MMP-2 expression was detected (data not shown). Taken together, our results suggest that downregulation of GnT-V disturbed the levels of EGFR, E-cadherin/vimentin and MMP-9 expression, thereby subsequently inhibiting the process of metastasis and invasion.

\section{Discussion}

$\mathrm{N}$-acetylglucosaminyltransferase V (GnT-V) catalyzes $\beta 1-6$ branching of $\mathrm{N}$-acetylglucosamine on asparagine-linked oligosaccharides of cell proteins $(21,32,33)$. The roles of GnT-V in the metastasis/invasion of various types of tumors are conflicting. Upregulated GnT-V activity has been reported in human colon cancer, hepatocarcinoma and breast cancer tissues $(6-8,34)$. However, increased GnT-V activity has been found to be associated with favorable stages as well as favorable prognosis in non-small cell lung cancers, bladder carcinomas and neuroblastoma (9-11). In the present study, we investigated the role of GnT-V in gastric cancer during the process of metastasis and invasion. The BGC823/GnT-V, BGC823, BGC823/ $\mathrm{NC}$ and BGC823/lipo cells were cultured for assaying. It was found that downregulation of GnT-V expression was accompanied by a reduction in $\beta 1-6 \mathrm{~N}$-acetylglucosamine branches, inhibition of cell growth and enhanced cell apoptosis induced by ATRA, resulting in inhibition of BGC823/GnT-V cell proliferation in vitro. Furthermore, the metastatic and invasive potential of BGC823/GnT-V cells was less than that of the other cell groups. These results demonstrated that targeted 

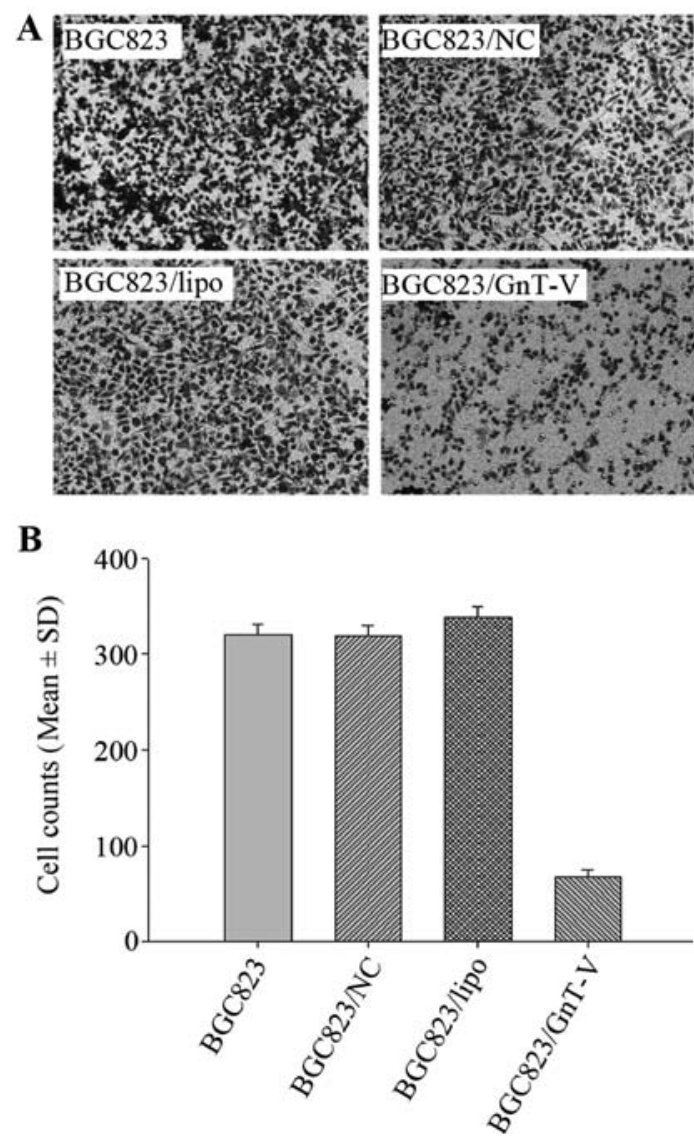

Figure 6. Invasion ability assay. (A) The invasive ability of cells was assesssed using 24 -well Transwell units with $8-\mu \mathrm{m}$ pore size polycarbonate inserts, and Matrigel as a basement membrane. After being cultured under the action of an inducer (cultured medium RMPI-1640 with 20\% FBS) for $48 \mathrm{~h}$, cells penetrating through the Matrigel-coated membrane were fixed by paraformaldehyde, stained with Giemsa reagent and counted in five different fields. These fields were selected randomly (magnification, x100). (B) The number of migrated cells are displayed on a bar diagram.

suppression of GnT-V reduced proliferation and invasion ability of BGC823 cells in vitro.

It is well known that EGFR-mediated signaling plays a crucial role in the control of cell metastasis and invasion in epithelial and cancer cells. To examine whether EGFRs are also required in the process of gastric cancer metastasis and invasion, EGFR, ErbB1, ErbB2 and ErbB3 levels were evaluated in BGC823 cells. The results indicated that suppression of GnT-V may decrease EGFR and ErbB2 gene transcript activity by reducing glycosylation of the transcription factors involved. Further investigation may be useful to answer this problem in the future. The decreased expression of ErbB4 at the protein level was induced by reduction of $\beta 1-6$ GlcNAc branching accompanied by GnT-V suppression as hypothesized before. As EGFR signaling is essential for tumor cell proliferation and migration/invasion, we assumed that downregulation of GnT-V inhibits proliferation and migration/invasion of gastric cancer cells by modulated EGFR signaling.

On the other hand, modification of E-cadherin N-glycans by GnT-V plays a role in tumor metastasis, as GnT-V has been reported to delocalize E-cadherin to the cytoplasm by posttranscriptional modification of E-cadherin $(35,36)$. However, little is known about the post-transcriptional modifications of
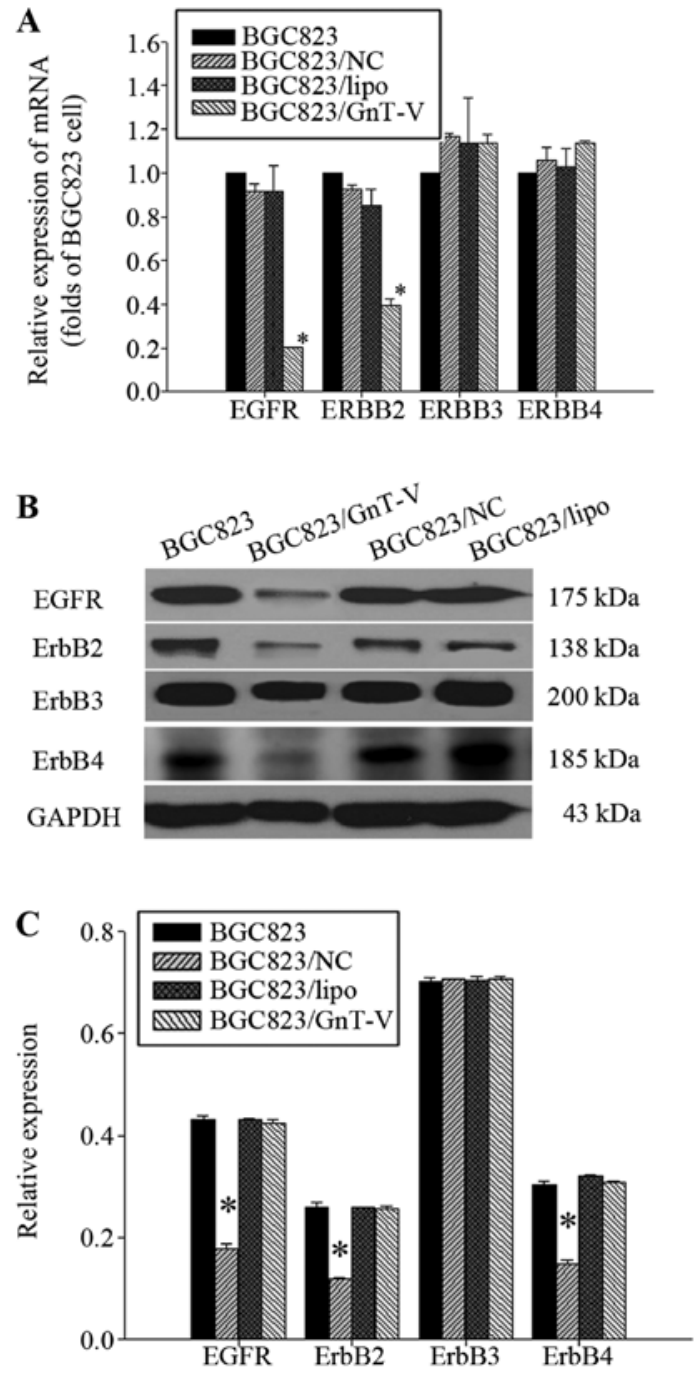

Figure 7. Expression of EGFRs. (A) Effect of the downregulation of GnT-V on the mRNA expression of EGFRs was determined by qRT-PCR. The mRNA expression of EGFR and ErbB2 in BGC823/GnT-V cells was decreased respectively, by $81.07 \pm 0.06$ and $60.50 \pm 1.19 \%$ when compared to the BGC 823 cells $(\mathrm{P}<0.05)$. The mRNA expression of ErbB3 and ErbB4 in BGC823/GnT-V, BGC823, BGC823/NC and BGC823/lipo cells had no significant differences $(\mathrm{P}>0.05)$. (B) EGFR, ErbB2, ErbB3 and ErbB4 protein expression in cells was determined by western blot assay. (C) Protein bands were quantified by Quantity One. The densitometric value of each protein band was normalized to GAPDH. The result are displayed on a bar diagram. The EGFR, ErbB2 and ErbB4 protein levels in BGC823/GnT-V cells when compared with the BGC823 cells were decreased by $55.21 \pm 0.01,50.54 \pm 0.00$ and $47.02 \pm 0.19 \%$, respectively $(\mathrm{P}<0.05)$. The expression of ErbB3 protein in BGC823/GnT-V, BGC823, BGC823/NC and BGC823/lipo cells had no significant differences $(\mathrm{P}>0.05)$. "Statistical significant.

E-cadherin and its role in E-cadherin-mediated tumor progression in gastric cancer cells. A substantial body of evidence has appeared to support the view that the E-cadherin function may mainly be affected by mechanisms through $\mathrm{N}$-glycosylation at the post-translational level in some carcinomas (37-39). However, our data demonstrated that downregulation of GnT-V affected the transcripts of E-cadherin and vimentin, and further influenced the protein expression of E-cadherin and vimentin.

The MMP family is considered to be one of the most important factors in tumor invasion. Particularly, the high expression of MMP-9 is considered as a crucial factor for 

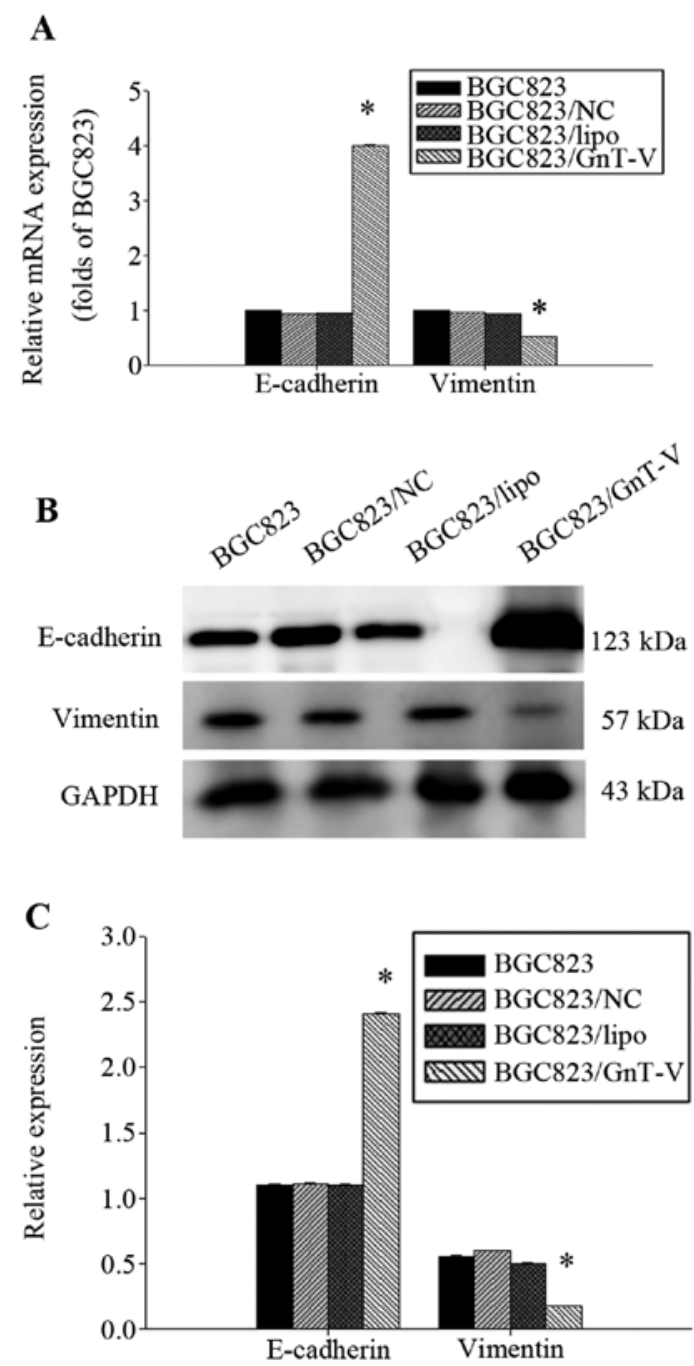

Figure 8. Expression of E-cadherin and vimentin. (A) Effect of the downregulation of GnT-V on the mRNA expression of E-cadherin and vimentin was determined by qRT-PCR. E-cadherin mRNA expression in the BGC823/ GnT-V cells was increased by $400.00 \pm 0.13 \%$ when compared to BGC823 cells $(\mathrm{P}<0.05)$. The vimentin mRNA expression in the BGC823/GnT-V cells was decreased by $47.15 \pm 0.01 \%$ when compared to that of the BGC 823 cells $(\mathrm{P}<0.05)$. (B) The E-cadherin and vimentin protein expression in cells was determined by western blot assay. (C) Protein bands were quantified by Quantity One. The densitometric value of each protein band was normalized to GAPDH. The results are displayed on a bar diagram. The E-cadherin protein expression in BGC823/GnT-V cells was increased by $118.25 \pm 0.07 \%$ when compared to that in the BGC823 cells $(\mathrm{P}<0.05)$. The vimentin protein expression in BGC823/GnT-V cells was decreased by $68.76 \pm 0.10 \%$ when compared to that in the BGC823 cells $(\mathrm{P}<0.05)$. "Statistically significant.

cell migration/invasion of endothelial cells into the adjacent stroma (30). It is essential to elucidate the molecular mechanisms underlying the $\mathrm{N}$-glycan regulation of the invasive function of MMP-9 in gastric cancer biology. The present study showed that MMP-9 expression in gastric cancer was decreased.

In conclusion, downregulation of the GnT-V gene inhibits invasion of gastric cancer BGC823 cells in vitro. The underlying mechanisms may be linked to EGFR signaling-initiated EMT phenotype and MMP-9 expression. These findings suggest that GnT-V may be a potential target for predicting the invasive potential of gastric cancer.
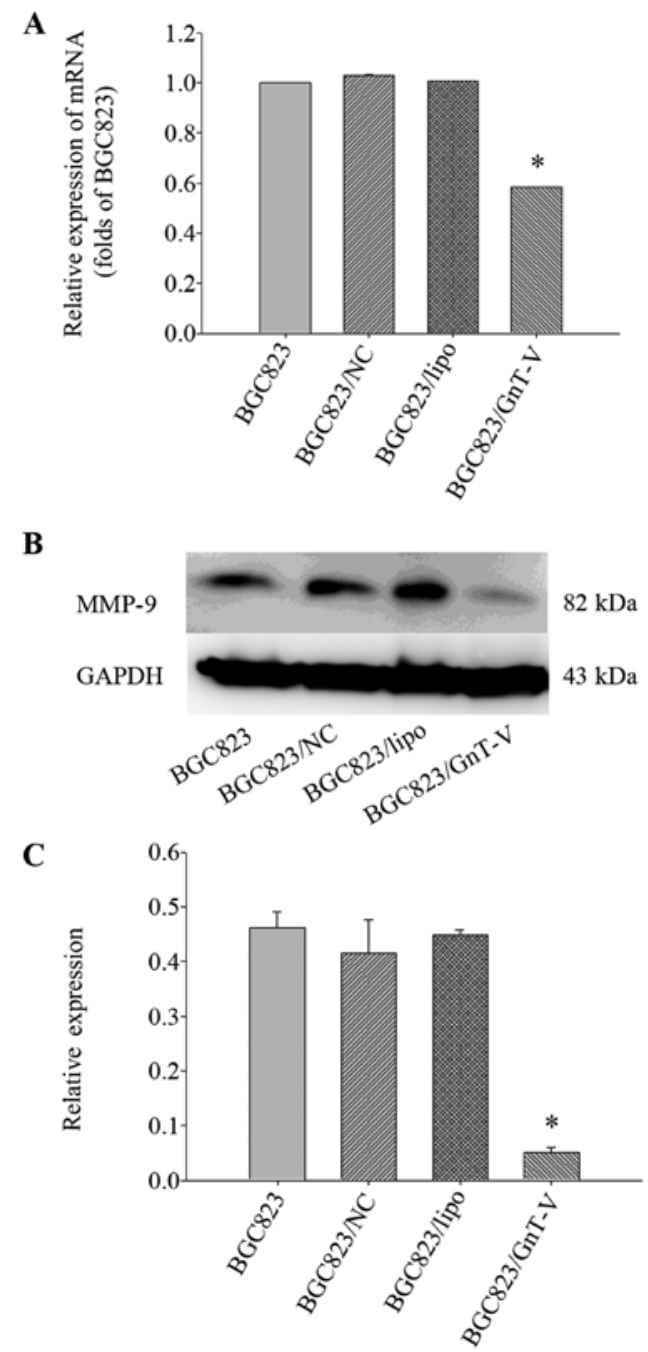

Figure 9. Expression of MMP-9. (A) Effect of the downregulation of GnT-V on the mRNA expression of MMP-9 was determined by qRT-PCR. The MMP-9 mRNA expression in BGC823/GnT-V cells was decreased by $41.76 \pm 0.01 \%$ when compared to the BGC823 cells $(\mathrm{P}<0.05)$. (B) The MMP-9 protein expression in cells was determined by western blot assay. (C) Protein bands were quantified by Quantity One. The densitometric value of each protein band was normalized to GAPDH. The results are displayed on a bar diagram. The MMP-9 protein expression was decreased in the BGC823/ GnT-V cells by $88.99 \pm 0.21 \%$ when compared to that in the BGC823 cells $(\mathrm{P}<0.05)$. *Statistically significant.

\section{Acknowledgements}

The present study was supported by a grant from the National Science Foundation of China (no. 81170333) and the Shanghai Committee of Science and Technology Key Projects for Basic Research (no. 12JC1408402).

\section{References}

1. Jemal A, Bray F, Center MM, Ferlay J, Ward E and Forman D: Global cancer statistics. CA Cancer J Clin 61: 69-90, 2011.

2. Nagini S: Carcinoma of the stomach: a review of epidemiology, pathogenesis, molecular genetics and chemoprevention. World $\mathbf{J}$ Gastrointest Oncol 4: 156-169, 2012.

3. Danaei G, Vander Hoorn S, Lopez AD, Murray CJ and Ezzati M; Comparative Risk Assessment collaborating group (cancers): Causes of cancer in the world: comparative risk assessment of nine behavioural and environmental risk factors. Lancet 366 : 1784-1793, 2005. 
4. Catalano V, Labianca R, Beretta GD, Gatta G, de Braud F and Van Cutsem E: Gastric cancer. Crit Rev Oncol Hematol 71: 127-164, 2009

5. Przybyło M, Pocheć E, Link-Lenczowski P and Lityńska A: Beta 1-6 branching of cell surface glycoproteins may contribute to uveal melanoma progression by up-regulating cell motility. Mol Vis 14: 625-636, 2008.

6. Murata K, Miyoshi E, Kameyama M, et al: Expression of $\mathrm{N}$-acetylglucosaminyltransferase $\mathrm{V}$ in colorectal cancer correlates with metastasis and poor prognosis. Clin Cancer Res 6: $1772-1777,2000$.

7. Guo P, Chen HJ, Wang QY and Chen HL: Downregulation of $\mathrm{N}$-acetylglucosaminyltransferase $\mathrm{V}$ facilitates all-trans retinoic acid to induce apoptosis of human hepatocarcinoma cells. Mol Cell Biochem 284: 103-110, 2005.

8. Xu YY, Lu Y, Fan KY and Shen ZH: Apoptosis induced by all-trans retinoic acid in $\mathrm{N}$-acetylglucosaminyltransferase $\mathrm{V}$ repressed human hepatocarcinoma cells is mediated through endoplasmic reticulum stress. J Cell Biochem 100: 773-782, 2007.

9. Dosaka-Akita H, Miyoshi E, Suzuki O, Itoh T, Katoh H and Taniguchi N: Expression of $\mathrm{N}$-acetylglucosaminyltransferase $\mathrm{V}$ is associated with prognosis and histology in non-small cell lung cancers. Clin Cancer Res 10: 1773-1779, 2004

10. Ishimura $\mathrm{H}$, Takahashi $\mathrm{T}$, Nakagawa $\mathrm{H}$, et al: $\mathrm{N}$-acetylglucsaminyltransferase $\mathrm{V}$ and beta1-6 branching N-linked oligosaccharides are associated with good prognosis of patients with bladder cancer. Clin Cancer Res 12: 2506-2511, 2006.

11. Inamori $\mathrm{K}, \mathrm{Gu} \mathrm{J}$, Ohira M, et al: High expression of N-acetylglucosaminyltransferase $\mathrm{V}$ in favorable neuroblastomas: involvement of its effect on apoptosis. FEBS Lett 580: 627-632, 2006.

12. Tian H, Miyoshi E, Kawaguchi N, et al: The implication of $\mathrm{N}$-acetylglucosaminyltransferase $\mathrm{V}$ expression in gastric cancer. Pathobiology 75: 288-294, 2008.

13. Hsieh CY, Tsai PC, Tseng CH, Chen YL, Chang LS and Lin SR Inhibition of EGF/EGFR activation with naphtho(1,2-b)furan4,5-dione blocks migration and invasion of MDA-MB-231 cells Toxicol In Vitro 27: 1-10, 2013.

14. Baselga $\mathbf{J}$ and Arteaga CL: Critical update and emerging trends in epidermal growth factor receptor targeting in cancer. J Clin Oncol 23: 2445-2459, 2005.

15. Rothhut B, Ghoneim C, Antonicelli F and Soula-Rothhut M: Epidermal growth factor stimulates matrix metalloproteinase- 9 expression and invasion in human follicular thyroid carcinoma cells through focal adhesion kinase. Biochimie 89: 613-624, 2007.

16. Mascia F, Cataisson C, Lee TC, et al: EGFR regulates the expression of keratinocyte-derived granulocyte/macrophage colony-stimulating factor in vitro and in vivo. J Invest Dermatol 130: 682-693, 2010

17. Pastore S, Mascia F, Mariani V and Girolomoni G: The epidermal growth factor receptor system in skin repair and inflammation. J Invest Dermatol 128: 1365-1374, 2008.

18. Pastore S and Mascia F: Novel acquisitions on the immunoprotective roles of the EGF receptor in the skin. Expert Rev Dermatol 3: 525-527, 2008

19. Kimura A, Terao M, Kato A, et al: Upregulation of N-acetylglucosaminyltransferase-V by heparin-binding EGF-like growth factor induces keratinocyte proliferation and epidermal hyperplasia. Exp Dermatol 21: 515-519, 2012.

20. Zhao Y, Sato Y, Isaji T, et al: Branched N-glycans regulate the biological functions of integrins and cadherins. FEBS J 275: 1939-1948, 2008.

21. Gu J, Zhao Y, Isaji T, et al: Beta1,4-N-acetylglucosaminyltransferase III down-regulates neurite outgrowth induced by costimulation of epidermal growth factor and integrins through the Ras/ERK signaling pathway in PC12 cells. Glycobiology 14: 177-186, 2004.

22. Zhao $\mathrm{Y}$, Nakagawa $\mathrm{T}$, Itoh $\mathrm{S}$, et al: $\mathrm{N}$-acetylglucosaminyl transferase III antagonizes the effect of $\mathrm{N}$-acetylglucosaminyl transferase $\mathrm{V}$ on $\alpha 3 \beta 1$ integrin-mediated cell migration. J Biol Chem 281: 32122-32130, 2006
23. Chavez MG, Buhr CA, Petrie WK, Wandinger-Ness A, Kusewitt DF and Hudson LG: Differential downregulation of E-cadherin and desmoglein by epidermal growth factor. Dermatol Res Pract 2012: 309587, 2012.

24. Terao M, Ishikawa A, Nakahara S, et al: Enhanced epithelial-mesenchymal transition-like phenotype in $\mathrm{N}$-acetylglucosaminyltransferase $\mathrm{V}$ transgenic mouse skin promotes wound healing. J Biol Chem 286: 28303-28311, 2011.

25. Zeisberg $M$ and Neilson EG: Biomarkers for epithelial-mesenchymal transitions. J Clin Invest 119: 1429-1437, 2009.

26. Dilly M, Hambruch N, Haeger JD and Pfarrer C: Epidermal growth factor (EGF) induces motility and upregulates MMP-9 and TIMP-1 in bovine trophoblast cells. Mol Reprod Dev 77: 622-629, 2010.

27. Delassus GS, Cho H, Park J and Eliceiri GL: New pathway links from cancer-progression determinants to gene expression of matrix metalloproteinases in breast cancer cells. J Cell Physiol 217: 739-744, 2008.

28. Fink K and Boratyński J: The role of metalloproteinases in modification of extracellular matrix in invasive tumor growth, metastasis and angiogenesis. Postepy Hig Med Dosw (Online) 66: 609-628, 2012 (In Polish).

29. Partridge JJ, Madsen MA, Ardi VC, Papagiannakopoulos T, Kupriyanova TA, Quigley JP and Deryugina EI: Functional analysis of matrix metalloproteinases and tissue inhibitors of metalloproteinases differentially expressed by variants of human HT-1080 fibrosarcoma exhibiting high and low levels of intravasation and metastasis. J Biol Chem 282: 35964-35977, 2007.

30. Di Carlo A: Matrix metalloproteinase-2 and -9 in the sera and in the urine of human oncocytoma and renal cell carcinoma. Oncol Rep 28: 1051-1056, 2012.

31. Zhuo E, He J, Wei T, et al: Down-regulation of GnT-V enhances nasopharyngeal carcinoma cell CNE-2 radiosensitivity in vitro and in vivo. Biochem Biophys Res Commun 424: 554-562, 2012.

32. Beheshti Zavareh R, Sukhai MA, Hurren R, et al: Suppression of cancer progression by MGAT1 shRNA knockdown. PLoS One 7: e43721, 2012

33. Xu Q, Isaji $\mathrm{T}, \mathrm{Lu} \mathrm{Y}$, et al: Roles of $\mathrm{N}$-acetylglucosaminyltransferase III in epithelial-to-mesenchymal transition induced by transforming growth factor $\beta 1$ (TGF- $\beta 1$ ) in epithelial cell lines. J Biol Chem 287: 16563-16574, 2012.

34. Li D, Li Y, Wu X, et al: Knockdown of Mgat5 inhibits breast cancer cell growth with activation of $\mathrm{CD}^{+}{ }^{+} \mathrm{T}$ cells and macrophages. J Immunol 180: 3158-3165, 2008.

35. Pinho SS, Reis CA, Paredes J, et al: The role of N-acetylglucosaminyltransferase III and $\mathrm{V}$ in the post-transcriptional modifications of E-cadherin. Hum Mol Genet 18: 2599-2608, 2009.

36. Pinho SS, Seruca R, Gärtner F, Yamaguchi Y, Gu J, Taniguchi $\mathrm{N}$ and Reis CA: Modulation of E-cadherin function and dysfunction by N-glycosylation. Cell Mol Life Sci 68: 10111020,2011

37. Zhao H,Liang Y, Xu Z, et al: $\mathrm{N}$-glycosylation affects the adhesive function of E-Cadherin through modifying the composition of adherens junctions (AJs) in human breast carcinoma cell line MDA-MB-435. J Cell Biochem 104: 162-175, 2008.

38. Liu T, Zhang X, Shang M, et al: Dysregulated expression of Slug, vimentin, and E-cadherin correlates with poor clinical outcome in patients with basal-like breast cancer. J Surg Oncol 107: 188-194, 2013

39. Roberti MP, Arriaga JM, Bianchini M, et al: Protein expression changes during human triple negative breast cancer cell line progression to lymph node metastasis in a xenografted model in nude mice. Cancer Biol Ther 13: 1123-1140, 2012. 